\title{
Comparing Radiological Features of Pulmonary Tuberculosis with and without HIV Infection
}

Banafsheh Moradmand Badie, Mehran Mostaan, Mehran Izadi*, Maryam Akhlaghkhah Neda Alijani, and Mehrnaz Rasoolinejad Iranian Research Center for HIVIAIDS (IRCHA), Tehran University of Medical Sciences, Tehran, Iran

\begin{abstract}
Objectives: The aim of this study was to determine and compare the radiological appearance of pulmonary tuberculosis (PTB) in groups of patients with and without human immunodeficiency virus (HIV) infection in a main referral center for Infectious disease in Iran.
\end{abstract}

Materials and Methods: One hundred and nineteen six patients with and without HIV infection was referred to infectious disease department of Imam Khomeini hospital.

The diagnosis of PTB was made on the basis of clinical history, physical examination, Ziehl-Neelsen microscopy on three sputum samples and chest radiography. The following issues were recorded for each radiograph: the number of zones involved with consolidation, cavitation, pleural effusion, bronchopulmonary pattern of spread; milliary disease; hilar and mediastinal lymphadenopathy.

Results: Out of all participant $65 \%$ were male. The diffuse pulmonary involvement was more common in PTB / HIV confection group than PTB without HIV infection (27.7\% vs $12 \%)$ Milliary pattern on chest X- ray in PTB / HIV group was more common too ( $17 \%$ vs $4.7 \%)$. However, HIV positive patients less commonly had cavitary lesion ( $4.3 \%$ vs $20.1 \%)$. The presence of pleural effusion was less common in PTB/ HIV group (13\% vs $23.5 \%$ ) and hilar lymphadenopathy was more common (10.6 vs $7.4 \%)$ in this group.

Conclusion: This study suggests that the rate of atypical patterns of CXR in PTB patients are growing because of increased frequency of HIV infection as well as IDUs, the main reason for the altered radiographic appearance of PTB may be due to way of selecting patients from a referral centre of infectious disease in country.

Keywords: Radiological features; TB; HIV

\section{Introduction}

HIV is the strongest risk factor for developing tuberculosis (TB) disease in those with latent or new Mycobacterium tuberculosis infection especially in endemic country like Iran. The risk of developing TB is more than 40 times greater in people living with HIV than among those who do not have HIV infection.

Tuberculosis is one of the most common infections to arise in the course of HIV infection. The prevalence of HIV among TB patients is estimated $23.4 \%$ in Iran. Furthermore, other studies among HIV positive patients with TB reveal that tuberculosis can be a common opportunistic infection in HIV positive infected individuals in Iran with a high prevalence of pulmonary as well as extrapulmonary type and the complex clinical presentation of disease.

In most developing countries like Iran diagnosis of pulmonary tuberculosis depends on the clinical symptoms, chest radiography, tuberculin skin test and sputum smear examination for acid-fast bacilli (AFB) [1]

Among diagnostic tools usually used for diagnosis of pulmonary tuberculosis, the chest $\mathrm{x}$-ray plays an essential role, chiefly since it is widely available within the public health system, even in poor population. Moreover, the emergence of HIV pandemic is having profound effect on the epidemiology, clinical features, diagnosis and management of tuberculosis. HIV infection leads to increased frequency of atypical radiographic features in patients with PTB with a tendency towards the pattern of primary disease [2-9]. These features include an increased frequency of lymphadenopathy and pleural effusions, less typical disease and less cavitations.Further studies have described a spectrum of radiographic appearances with atypical features becoming more common with greater degrees of immunodeficiency as reflected by CD4+ T cell lymphopenia [10-12]. This study designed to compare the radiographic characteristics of patients with Pulmonary Tuberculosis (PTB) and Human Immunodeficiency Virus (HIV) infection with those of HIV negative patients.

\section{Materials and Methods}

The study was carried out at the infectious disease department of IMAM KHOMEINI hospital in Tehran, IRAN. The sample of 196 PTB patients with and without HIV infection was referred to infectious disease department of Imama Khomeini hospital from March 2004 to January 2007. The diagnosis of PTB was made on the basis of clinical history; physical examination besides all the patients had three sputum smear exams for acid fast bacilli using the Ziehl-Neelsen technique, moreover HIV infected patients were diagnosed with ELISA then approved with Western Blot test. The tuberculin test was performed with $0.1 \mathrm{ml}$ of PPD RT23, according to a standardized technique; the patients with TB infection were included in this study according positive sputum smear exam, positive history and positive chest $\mathrm{X}$-ray.

*Corresponding author: Mehran Izadi, Iranian Research Center for HIVIAIDS (IRCHA), Tehran University of Medical Sciences, Tehran, Iran, Tel: +98(21) 66947984; Fax: +98(21)66918899; E-mail: izadi2003@yahoo.co

Received July 16, 2012; Accepted November 27, 2012; Published November 30,2012

Citation: Badie BM, Mostaan M, Izadi M, Alijani MAN, Rasoolinejad M (2012) Comparing Radiological Features of Pulmonary Tuberculosis with and without HIV Infection. J AIDS Clinic Res 3:188. doi:10.4172/2155-6113.1000188

Copyright: ( 2012 Badie BM, et al. This is an open-access article distributed unde the terms of the Creative Commons Attribution License, which permits unrestricted use, distribution, and reproduction in any medium, provided the original author and source are credited. 


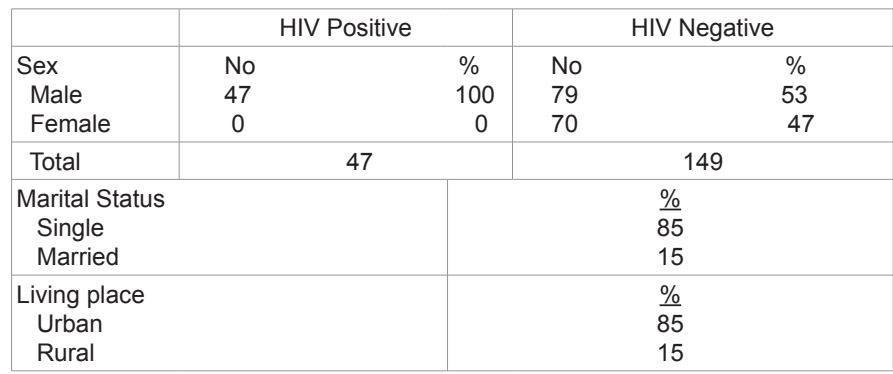

Table 1: Demographic characteristic of 196 pulmonary tuberculosis patients with mean age of 79 years.

\begin{tabular}{|lccc|}
\hline Radiological & TB/HIV & TB/HIV & P value \\
\hline Patterns & positive & Negative & \\
\hline Normal & $2.3 \%$ & $0 \%$ & \\
\hline $\begin{array}{l}\text { Focal pulmonary } \\
\text { consolidation }\end{array}$ & $38 \%$ & $51 \%$ & 0.0 \\
\hline $\begin{array}{l}\text { Diffuse pulmonary } \\
\text { Consolidation }\end{array}$ & $27.7 \%$ & $12 \%$ & 0.01 \\
\hline Milliary pattern & $17 \%$ & $4.7 \%$ & 0.01 \\
\hline Adenopathy & $10.6 \%$ & $7.4 \%$ & 0.54 \\
\hline Pleural effusion & $13 \%$ & $23.5 \%$ & 0.082 \\
\hline Pleural thickening & $0 \%$ & $4 \%$ & 0.0 \\
\hline Cavitation & $4.3 \%$ & $20.1 \%$ & 0.0 \\
\hline
\end{tabular}

Table 2: Radiological features of 196 pulmonary tuberculosis patients $\mathrm{HIV}^{+}$and $\mathrm{HIV}$.

The chest radiographs were analyzed by an expert radiologist. The radiographic features were summarized in categories. The following radiological features were found: the number and zones involved with consolidation, cavitation, pleural effusion, bronchopulmonary pattern of spread: milliary disease: hilar and mediastinal lymphadenopathy. The statistical analysis was performed using SPSS 17 (SPSS Inc., Chicago, IL, USA). Values were tested for normality besides $t$-test, chi square was used and $P$-value of 0.05 or less was considered significant as well.

The research was approved by the ethics committee of Tehran University of medical science.

\section{Results}

\section{Patients' characteristics}

Out of 196 patients with Tuberculosis 47 (24\%) were HIV positive and $149(76 \%)$ were HIV negative with mean age of 79 years. All HIV infected patients were 21 to 45 years old. Seventy five percent of all patients had positive sputum smear test. Table- 1 shows the demographic characteristic; besides $93.4 \%$ of HIV infected patients were intravenous drug user (IDU) whereas $13.2 \%$ of patients without HIV were IDU.

\section{Chest radiograph appearances}

One hundred ten out of 196 patients (57.7\%) were between 2145 years old, in this group the cavitation was less common in HIV positive patients Chest X-ray, compared with HIV negative ones ( $4.9 \%$ vs $27.7 \%$ ). Again in this group (21-45 years) diffuse pulmonary involvement was more common in HIV / PTB co-infected patients ( $24.3 \%$ vs $5.8 \%$; $\mathrm{P}=0.01$ ) than those with HIV negative.Miliary pattern was more common among HIV positive patients ( $17 \%$ vs $4.7 \%, \mathrm{P}=0.01$ ) than HIV negative ones. On the other hand, there was insignificant statistical trend towards less pleural effusion $(13 \%$ vs $23.5 \% ; \mathrm{P}=0.082)$ as well as hillar lymphadenopathy $(10.6 \%$ vs $7.4 \% ; \mathrm{P}=0.54)$ in seropositive cases. Table-2 illustrates the radiological findings of 196 pulmonary tuberculosis patients with and without HIV separately.

\section{Discussion}

Chest X-ray abnormalities are non-specific in HIV-infected patients than in HIV-negative patients, which may result in under diagnosis of tuberculosis in these patients. Radiological patterns depend on the level of immunity in the host.

Tuberculosis, unlike other HIV-associated opportunistic infections, may occur at any levels of immunosuppressant, although its frequency markedly increases in patients with more severe immunosuppression.

Diagnosis of PTB is based on chronic symptoms and chest X-ray changes and sputum smear and culture but definitive diagnosis can be difficult as clinical and radiological findings may be non-specific especially in HIV infected patients but in resource limited countries, most of pulmonary tuberculosis diagnosis is based on clinical features and chest X- ray [4].

In advanced HIV disease, the chest radiographic findings of pulmonary TB are markedly different compared with those among patients with less severe immunosuppression. Lower lobe, middle lobe, interstitial, and miliary infiltrates are common and cavitation is less common $[4,5]$.

The study shows that atypical radiological appearance of PTB in HIV positive subjects is common and mostly related to the low level of patients' immunity system, but some radiographic findings like pleural effusion were less common in our HIV positive patients compared with other studies. It should be considered that $93.3 \%$ of our HIV positive subjects were IDU but in other studies the rate of heterosexually acquired HIV infection was more common (60-80\%). So, it may be the reason of low rate pleural effusion among our patients in comparison to other studies. The various radiological features are caused by the effect of decreased cell-mediated immunity in patients with HIV infection. This impairment of cell-mediated tissue response could explain the relative paucity of consolidation and pleural thickening. The most characteristic sign of PTB in chest X-ray in patients without HIV is cavitations that were very rare in our patients.

This is the reflection of poor cell mediated immunity [1]. This may show us that our patients are in late stage of disease and PTB is one of opportunistic infections in very early and late onset of HIV infections.

Tuberculosis classically involves the upper lobes usually unilaterally. Among patients with HIV and pulmonary tuberculosis coinfection, bilateral lung involvement was observed among $27.7 \%$ bilateral consolidation, $17 \%$ miliary pattern, in TB /HIV versus among $12 \%$ pulmonary bilateral consolidation, $4.7 \%$ miliary pattern of the chest radiographsin HIV negative tuberculosis patients, suggesting that diffuse involvement is more common in patients with HIV and TB coinfection as in a study by Mahesha Padyana et al. [10].

In SD. Greenberg et al. [2] and R. Long et al. [3] studies the frequency of miliary pattern found between $2.3 \%$ to $8 \%$ whereas in this study the milliary appearance was $17 \%$ which may caused by selection of patients with more advanced immunosuppression $[2,3]$.

They reported lymphadenopathy pattern in HIV positive patients with PTB in East and Africa which was dramatically high in rate (30$50 \%$ ) pleural effusion $30-43 \%[8,9]$ and milliary pattern $6-8 \%[8,9,12]$ as compared with $10.6 \%, 13 \%$ and $17 \%$ respectively in this study. Our findings are somehow resemble to other studies in other countries, but unfortunately in other studies like ours there was no CD4 count for estimating the immune- suppression level. The most significant differences between our results with other studies were racial variation 
Citation: Badie BM, Mostaan M, Izadi M, Alijani MAN, Rasoolinejad M (2012) Comparing Radiological Features of Pulmonary Tuberculosis with and without HIV Infection. J AIDS Clinic Res 3:188. doi:10.4172/2155-6113.1000188

Page 3 of 3

in host response to $M$. tuberculosis which has a genotypic basis [10-17]. Chest X-ray abnormalities are even more non-specific in HIV-infected patients than in HIV-negative patients, which may result in under diagnosis.

\section{Conclusion}

This study indicated atypical radiological features in PTB associated with HIV Infection. Moreover, chest radiography still has recommended as a useful diagnostic test for PTB in HIV positive patients. Atypical presentations in any patient especially immunosuppressive subject, physicians should immediately check HIV as well. Although in present study there were no CD4 counts or Viral loads data, we truly recommend measuring CD4 counts and viral loads in patients with suspected radiological features.

In our study, most common radiological presentation of tuberculosis in HIV infected patients was diffuse pulmonary consolidation followed by miliary shadows Involvement of lung field in tuberculosis was atypical as evidenced by bilateral lung involvement; middle and lower zone or diffuse involvement. Comparison of $\mathrm{CD} 4$ count with chest $\mathrm{X}$-ray is very important for interpretation. Active tuberculosis is common among HIV-infected persons living in tuberculosis endemic countries like our country tuberculosis must be kept in our mind routinely with any chest $\mathrm{X}$ - ray as we see in our research.

\section{References}

1. Jamal LF, Moherdaui F (2007) Tuberculosis and HIV infection in Brazil: magnitude of the problem and strategies for control. Rev Saúde Pública 41: 104-110.

2. Greenberg SD, Frager D, Suster B, Walker S, Stavropoulos C, et al. (1994) Active pulmonary tuberculosis in patients with AIDS: spectrum of radiographic findings (Including a normal appearance). Radiology 193: 115-119.

3. Long R, Maycher B, Scalcini M, Manfreda J (1992) The chest roentgenogram in pulmonary tuberculosis patients seropositive for human immunodeficiency virus type I. Chest 99: 123-127.

4. Guidelines for Prevention and Treatment of Opportunistic Infections in HIVInfected Adults and Adolescents Morbidity and Mortality. Weekly Report 58: RR-4.
5. (2011) Tuberculosis National Institute for Health and Clinical Excellence.

6. Elliott AM, Halwiindi B, Hayes RJ, Luo N, Tembo G, et al. (1993) The impact of human immunodeficncy virus on the presentation and diagnosis of tuberculosis in a cohort study in zambia. J Trop med hyg 96: 1-11.

7. Bakari M, Arbeit RD, Mtei L, Lyimo J, Waddell R, et al. (2008) Basis for treatment of tuberculosis among HIV-infected patients in Tanzania: the role of chest x-ray and sputum culture. BMC Infect Dis 8: 32.

8. Noronha D, Pallangyo KJ, Ndosi BN, Lweno H, Sabuka SR (1991) Radiological features of pulmonary tuberculosis in patients infected with human immunodeficiency virus. East Aft Med J 68: 210-215.

9. Saks AM, Posner R (1992) Tuberculosis in HIV positive patients in South Africa: a comparative radiological study with HIV negative patients. Clin Radiol 46 : 387-390.

10. Padyana M, Bhat RV, Dinesha M, Nawaz A (2012) HIV-Tuberculosis: A Study of Chest X-Ray Patterns in Relation to CD4 Count. N Am J Med Sci 4: 221-225.

11. Keiper MD, Beumont M, Elshami A, Langlotz CP, Miller WT Jr (1995) CD4 lymphocyte count and the radiographic presentation of pulmonary tuberculosis. A study of the relationship between these factors in patients with human immunodeficiency virus infection. Chest 107: 74-80.

12. Post FA, Wood R, Pillay GP (1995) Pulmonary tuberculosis in HIV infection radiologic appearance is related to $C D+T$-lymphocyte count. Tuberc Lung Dis 76: 518-521.

13. Ahmad Z, Shameem M (2005) Manifestation of tuberculosis in HIV infected patients. JIACM 6: 302-305

14. Prasad R, Saini JK, Gupta R, Kannaujia RK, Sarin S, et al. (2004) A comparative study of Clinico-radiological spectrum of tuberculosis among HIV Seropositive and HIV seronegative patients. Indian J Chest Dis Allied Sci 46: 99-103

15. Mabiala Babela JR, Makosso E, Senga P (2006) Radiological Specify of pulmonary tuberculosis in Congolese children: effect of HIV infection. Med Trop 66: 255-259.

16. Awoyemi OB, Ige OM, Onadeko BO (2002) Pattern of active pulmonary tuberculosis in human immunodeficiency virus seropositive adult patients in University College Hospital, Ibadan, Nigeria. Afr J Med Med Sci 31: 25-31.

17. Bellamy R, Ruwende C, Corrah T, McAdam KP, Whittle HC, et al. (1998) variations in the NRAMPI gene and susceptibility to tuberculosis in West Africans. N Engl J Med 338: 640-644. 